\title{
Pressure Sensor Catheter based on Micromachined Aluminum Nitride Membrane Fiber Tip Fabry-Pérot-Interferometer
}

\author{
Marvin Friedemann \\ Department of Electrical \\ Engineering and \\ Information Technology \\ Chemnitz University of \\ Technology \\ Chemnitz, Germany \\ marvin.friedemann@ \\ etit.tu-chemnitz.de \\ Katja Meinel \\ Department of Electrical \\ Engineering and \\ Information Technology \\ Chemnitz University of \\ Technology \\ Chemnitz, Germany
}

\author{
Sebastian Voigt \\ Department of Electrical \\ Engineering and \\ Information Technology \\ Chemnitz University of \\ Technology \\ Chemnitz, Germany \\ sebastian.voigt@ \\ etit.tu-chemnitz.de \\ Raoul Hecker \\ Specialist for Public \\ Health and Occupational \\ Medicine
}

\author{
David Kriebel \\ Department of Electrical \\ Engineering and \\ Information Technology \\ Chemnitz University of \\ Technology \\ Chemnitz, Germany
}

Karla Hiller

Department of Electrical

Engineering and

Information Technology

Chemnitz University of

Technology

Chemnitz, Germany

\author{
Chris Stöckel \\ Department of Electrical \\ Engineering and \\ Information Technology \\ Chemnitz University of \\ Technology \\ Chemnitz, Germany
}

Jan Mehner

Department of Electrical Engineering and Information Technology

Chemnitz University of Technology

Chemnitz, Germany

\begin{abstract}
This paper introduces an absolute pressure sensor based on an extrinsic fiber-optic Fabry-Pérot-Interferometer molded into a silicone catheter. The interferometer principle is formed between the tip of an optical fiber and a reflective multilayer membrane containing aluminum nitride. The signal analysis is done by a Fiber Bragg Grating interrogation device. First, the optical sensing principle as pressure sensor in an invasive catheter with a diameter of $1 \mathrm{~mm}$ is described. Then, the dependence of the optical signal on changes of hydrostatic pressure and ambient temperature is shown. Out of these characteristics it was possible to evaluate the pressure sensor catheter for potential application in the human vascular system.
\end{abstract}

Keywords- Fiber-optic sensors, Fabry-Pérot-Interferometer, hydrostatic pressure, temperature, interrogation device, AlN membrane, catheter

\section{INTRODUCTION}

Due to their variety of advantages, fiber-optic sensors offer a large scope in measurements of strain, pressure, temperature, and other parameters. Among them, Fiber Bragg Gratings (FBG) and Fiber-optic Fabry-Pérot-Interferometer (FFPI) offer multiplexing, signal linearity, small size and especially electromagnetic compatibility due to the nonelectric sensing principle. Hence, they are particularly appropriate for medical applications in measurement catheters [1-3]. An integration of an FBG sensor array along a catheter for the use in the human intestine can diagram peristalsis as pressure profile, developed by Arkwrigth et al. [4, 5]. Further, Poeggel et al. presented a catheter for pressure measurements in urodynamics [6, 7]. FBG and FFPI could also be conceivable in application fields of spinal stenosis or invasive blood pressure measurements. Especially for the purpose of blood circulation recordings the diameter is significant for the positioning of the probe. Catheter for coronary arteries should have max. 3 Fr $(1 \mathrm{~mm})$ diameter. This paper introduces a pressure sensor catheter with a diameter of almost $1 \mathrm{~mm}$. The basis of the pressure catheter is an optical silicate glass fiber with a diameter of $125 \mu \mathrm{m}$, comprising core and cladding. On the fiber tip, a micromachined round silicon chip with an outer diameter of about $600 \mu \mathrm{m}$ and a flexible multilayer membrane containing aluminum nitride (AlN) builds a cavity for absolute pressure sensing. According to [8], AlN membranes show excellent characteristics in non-invasive pulse wave measurements. Both components are coated with medical silicone over a flexible length up to $1.5 \mathrm{~m}$. An FBG interrogation device detects the interference pattern due to light reflection on the AlN membrane. Our LabView-software is able to evaluate the changes in the interference spectrum as a consequence of pressure or temperature changes. The use of an FBG interrogation device for evaluation is a good opportunity for promising results in further developments of FBG-FFPI-hybrid simultaneous sensors, opening new fields of applications for fiber-optic sensors.

\section{DESIGN AND PRINCIPLE}

\section{A. Reflective Membrane}

The fiber tip and the reflective membrane form an FFPI. This membrane is part of a piezoelectric pressure sensor chip. Due to piezoelectric effect of the AlN layer the membrane can be actuated. In this paper it is used as passive membrane in order to demonstrate its passive behavior. We plan to use it in a closed loop mode because this has many advantages in the readout technique. The pressure sensor chip is circular shaped with an outer diameter of $600 \mu \mathrm{m}$, a membrane diameter of $200 \mu \mathrm{m}$ and a total height of $400 \mu \mathrm{m}$. On the backside of the chip there is a hole of $200 \mu \mathrm{m}$ diameter. This is used to hold the optical fiber, which is placed centrally on the bottom side. Fig. 1 shows the schematic section of the membrane. It consists of $1 \mu \mathrm{m}$ thermal oxide $\left(\mathrm{SiO}_{2}\right), 100 \mathrm{~nm}$ physical vapor deposition platinum and $600 \mathrm{~nm}$ AlN grown from the platinum layer which acts as seed layer and contact layer at same time. Top electrodes are formed by aluminum (Al) [9]. With this small layer thickness, the membrane deflects depending on hydrostatic pressure [9]. Due to the reflective properties of platinum and $\mathrm{SiO}_{2}$ respectively, the pressure sensor may also be used as passive, pressure depending FFPI cavity without electrical actuation. The presented catheter works with this passive principle and has the advantage of not taking electrical wires into account. Therefore, a smaller catheter diameter is achievable. The bonding between optical fiber and pressure sensor chip is displayed in Fig. 2. 


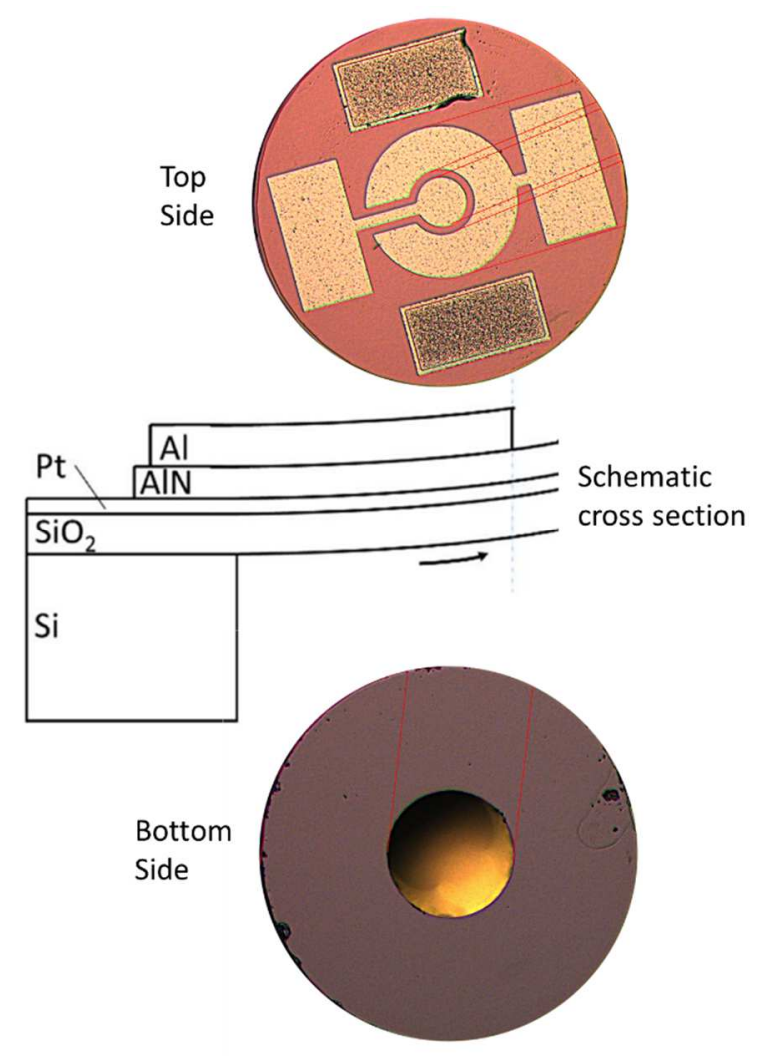

Fig. 1. Cross section (schematic), top and bottom view of the pressure sensor chip.

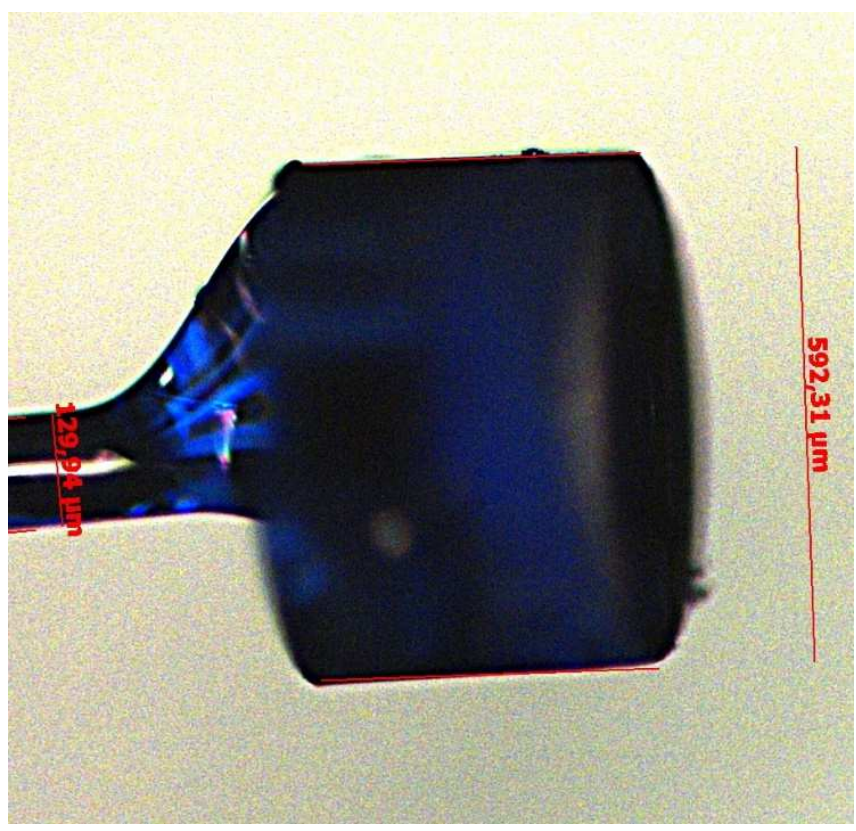

Fig. 2. Pressure sensor chip and optical fiber tip with both wetting epoxy resin.

\section{B. Fabry-Pérot-Interferometer}

A Fabry-Pérot-Interferometer (FPI) is a phase-modulated optical measuring instrument, typically consisting of two reflective planes. The space between both planes is called cavity. Induced light produces interference due to the superposition of the light waves as a consequence of optical path difference (OPD). The OPD describes the length of the cavity. The shape of the interference pattern varies depending on the value of the OPD [10].
An FFPI describes the assembling of an optical fiber and an FPI. A distinction is made in the realization of the cavity. Therefore, an intrinsic FPI has the cavity within the fiber (e.g. due to etching or laser processes). An extrinsic FPI is the aggregation of a reflective membrane outside the optical fiber in front of the fiber tip, as introduced in our pressure sensor catheter. The cavity is realized between the fiber tip (light reflection and transmission) and the reflective membrane [11].

We observe that changes in hydrostatic pressure and ambient temperature result in shifts of the wavelength peaks in the interference pattern. Fig. 3 shows the evaluation principle. The initial interference pattern is shifted when applying hydrostatic pressure on the FFPI. We use the mean value of the peak shifts from the highest five maxima $\Delta \lambda p$ as measure for hydrostatic pressure changes and for calibration of the pressure sensor because of the almost consistent relative wavelength shift of each maxima. Other possibilities of the interference pattern signal evaluation are Two-PointInterrogation (distance between two maxima proportional to OPD variation) and Fourier-Transformation (changes in frequency of the periodic pattern due to OPD variations) [12].

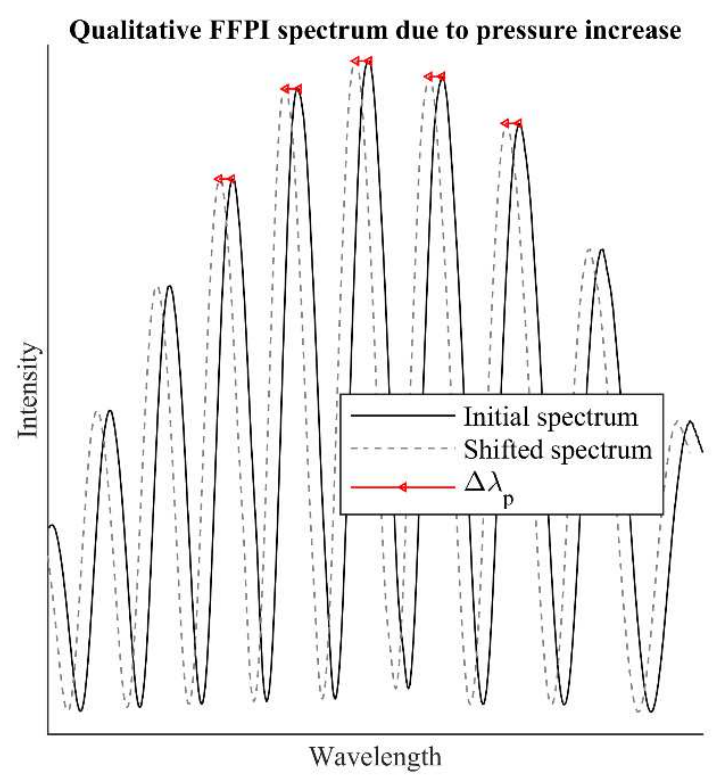

Fig. 3. Evaluation principle of an interference pattern with peak shift averaging of the highest five peaks.

\section{Signal processing}

The interrogation device comprises a superluminescent diode (SLED), a spectrometer (including polychromator and charge coupled device image sensor) and a drift prevention system (including a housing heating). Broadband light is coupled into the optical fiber. The SLED includes a circulator for transmitting the FFPI-signal to the spectrometer. The wavelength measurements and calculations of peak shifts are done with a LabView evaluation software. The optoelectronical components are heated up to a constant temperature for drift prevention in the measured intensity and wavelength evaluation [13].

\section{Fabrication and catheter assembly}

The optical fiber is placed orthogonal to the membrane, while controlling the interference pattern to prevent membrane damage through harsh contact with the fiber tip. Epoxy resin ensures a fixation at a stable position and a sealing 
of the cavity around the fiber in front of the tip. The FFPI is sealed with medical silicone to reach an outer diameter of about $3 \mathrm{Fr}(1 \mathrm{~mm})$. The catheter tip is shown in Fig. 4. The silicone thickness in front of the pressure sensor chip is about $1.3 \mathrm{~mm}$ for membrane protection.

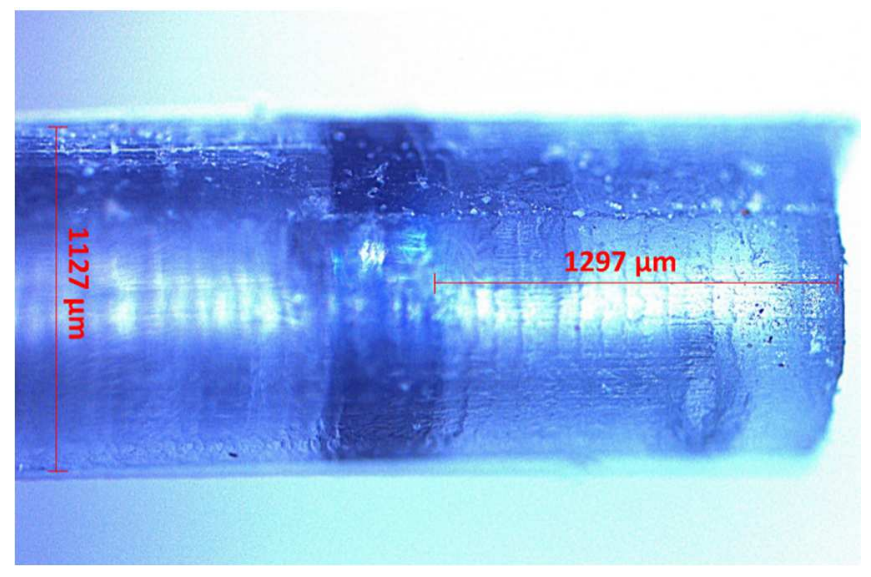

Fig. 4. Catheter tip with encapsulated pressure sensor chip and optical fiber.

\section{EXPERIMENTAL PROCEDURE}

\section{A. Measurement setup}

To characterize the pressure sensitivity, the catheter was placed on the bottom of a water column. With this setup it was possible to simulate hydrostatic pressure impact on the catheter tip. By means of manually operating valves, pressure fluctuations were adjusted. The change in reference hydrostatic pressure was measured via the water level. Knowing that $1 \mathrm{~cm}$ water level increase $(1 \mathrm{cmH} 2 \mathrm{O})$ applies a hydrostatic pressure rise of about $1 \mathrm{mbar}$, the wavelength shift due to pressure was determined. Our readout software uses the incoming intensity spectrum and calculates the wavelength shifts of each of the five highest peaks as well as the average value for evidence of an equal shift of each peak. Due to high computing effort the practicable scan rate was about $11 \mathrm{~Hz}$. The incoming FFPI spectrum, recorded under atmospheric standard air pressure, is plotted in Fig. 5.

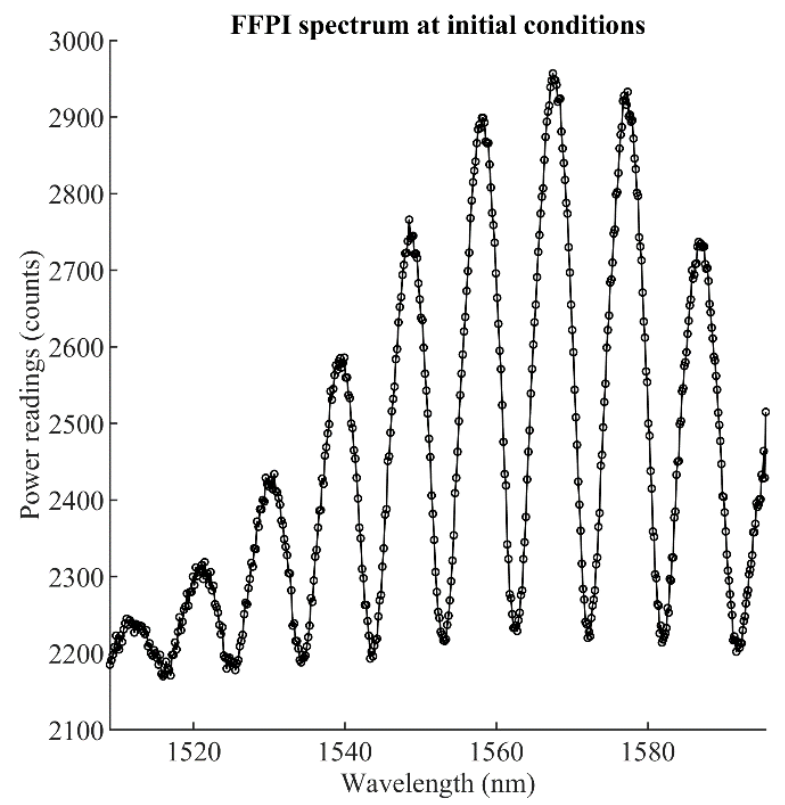

Fig. 5. Initial FFPI spectrum, every dot complies with a pixel on the image sensor.

\section{B. Pressure sensitivity}

Refilling the water column and subsequently draining it generates hydrostatic pressure ramps. Therefore, the ramp target pressure was raised in 10 mbar steps up to a total increase in pressure of approximately 96 mbar. The relative wavelength per peak shift is determined from the difference of the absolute wavelength values at initial water level (6 mbar) and top water level ( 6 mbar +10 mbar steps). By updating the initial pressure value per ramp cycle, the impact on the sensor due to low, not reliable measurable fluctuations in temperature of flowing water (e.g. slow mixing, temperature sensor inertia and resolution) was minimized.

The experimental results are plotted in Fig. 6. With rising hydrostatic water pressure the wavelength maxima values decrease. The diagram shows an almost linear relation between the relative shift of each chosen maxima wavelength in the FFPI spectrum and variation of hydrostatic pressure. Therefore, each single peak shift has almost the same increase. The relative average wavelength shift is almost $-0.008 \mathrm{~nm} / \mathrm{mbar}$. Due to the low standard deviation of about $0.0002 \mathrm{~nm}(\approx 2.7 \%)$ the functionality of the evaluation principle with peak shift measurement out of the FFPI spectrum is confirmed.

Regarding to minimal differences in peak detection a fluctuation margin of approximately $\pm 0.01 \mathrm{~nm}$ comes into effect. Hence, a total resolution of about 5 mbar seems realistic. With this value, blood pressure recordings with an accuracy of about $\pm 3.8 \mathrm{mmHg}$ are expectable. With the application of silicon chips with larger AlN membrane diameter an even higher pressure accuracy seems achievable.

Fig. 7 shows the average absolute wavelength of peak shift at absolute hydrostatic water column pressure values.

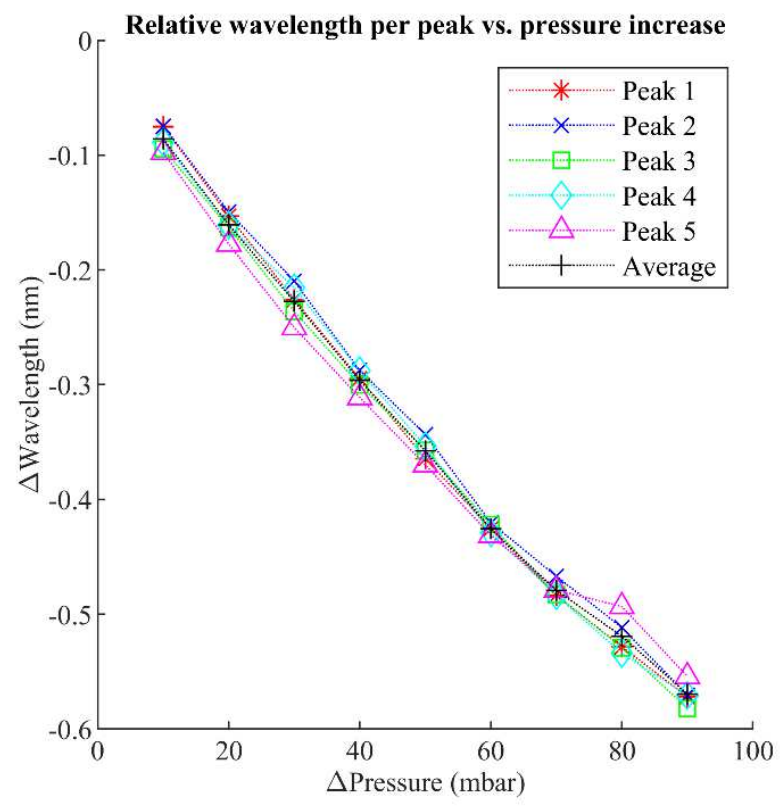

Fig. 6. Relative wavelength shift of the highest five peaks as well as their average due to pressure ramps. 


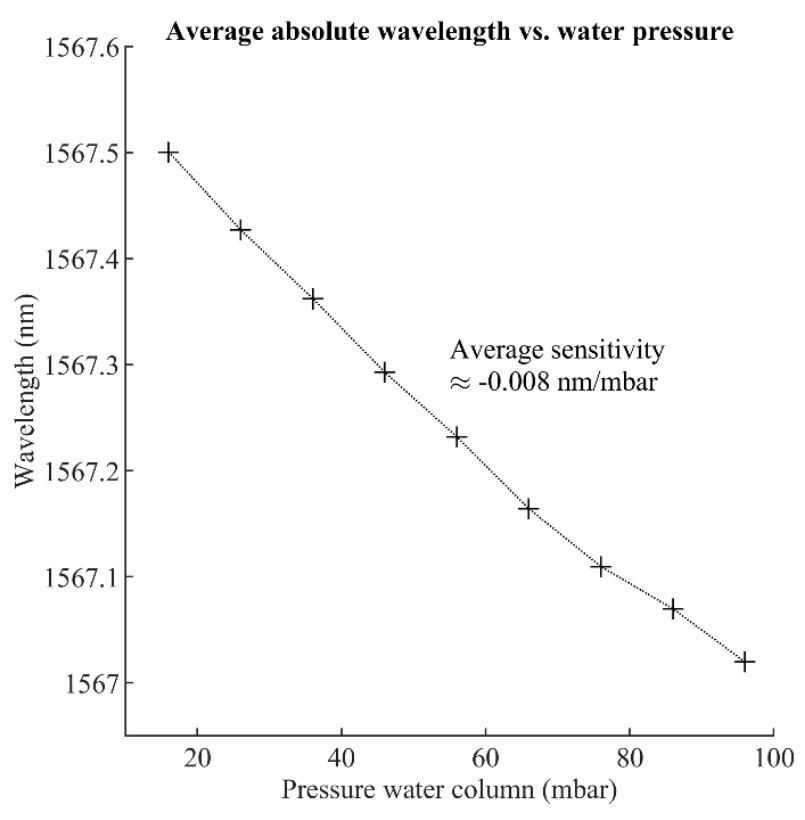

Fig. 7. Absolute wavelength shift of average peak values due to pressure increase with water column.

\section{Temperature sensitivity}

When applying the FFPI pressure sensor catheter at ambient with higher temperature fluctuations the pressure sensor's temperature dependency must be considered. Therefore, the catheter was placed in a heating chamber and impinged with a temperature ramp of up to $10 \mathrm{~K}$. The reference temperature was measured with a Pt1000 temperature sensor (resolution $0.1 \mathrm{~K}$ ). In this experiment, an almost linear relation between temperature and average wavelength shift was detected. The wavelength value increases with rising ambient temperature with a sensitivity of about $0.2 \mathrm{~nm} / \mathrm{K}$. The curve is not exact straight due to ventilator generated vibrations and air flows as well as temperature sensor resolution limitations.

\section{CONCLUSION}

We used a micromachined round silicon chip with an AIN membrane as a passive hydrostatic pressure sensor inside a $1 \mathrm{~mm}$ thick catheter tip. The device shows the road to very small all optic pressure sensor catheters with multiple sensing options. The absolute pressure at the tip is a key feature. Characterization revealed a linear dependency between the wavelength shift of an FFPI interference pattern which can be read out by standard FBG interrogation spectrometers. The wavelength shift was quantified to $-0.008 \mathrm{~nm} / \mathrm{mbar}$ with a membrane of $200 \mu \mathrm{m}$ diameter. The overall chip diameter was $600 \mu \mathrm{m}$. The temperature dependency is quantified to $0.2 \mathrm{~nm} / \mathrm{K}$ and needs to be compensated by a temperature measurement using an FBG in the same fiber. The simultaneous readout will be published soon by the authors.

\section{REFERENCES}

[1] A. Othonos and K. Kalli, Fiber Bragg gratings: Fundamentals and applications in telecommunications and sensing. Boston, Mass.: Artech House, pp. 95-174, 1999.

[2] S. Poeggel et al., "Recent Improvement of Medical Optical Fibre Pressure and Temperature Sensors," Biosensors, vol. 5, no. 3, pp. 432449, 2015.

[3] S. Poeggel, D. Tosi, D. Duraibabu, G. Leen, D. McGrath, and E. Lewis, "Optical Fibre Pressure Sensors in Medical Applications," Sensors (Basel, Switzerland), vol. 15, no. 7, pp. 17115-17148, 2015.

[4] J. W. Arkwright et al., "In-vivo demonstration of a high resolution optical fiber manometry catheter for diagnosis of gastrointestinal motility disorders," Optics express, vol. 17, no. 6, pp. 4500-4508, 2009.

[5] J. W. Arkwright et al., "Measurement of Muscular Activity Associated With Peristalsis in the Human Gut Using Fiber Bragg Grating Arrays," IEEE Sensors J., vol. 12, no. 1, pp. 113-117, 2012.

[6] D. Duraibabu et al., "Optical fibre pressure and temperature sensor system designed for urodynamic applications," in Sixth European Workshop on Optical Fibre Sensors, Limerick, Ireland, 2016, pp. 1-6.

[7] S. Poeggel et al., "Fiber-Optic EFPI Pressure Sensors for In Vivo Urodynamic Analysis,” IEEE Sensors J., vol. 14, no. 7, pp. 2335-2340, 2014.

[8] A. Bongrain et al., "A New Technology of Ultrathin AlN Piezoelectric Sensor for Pulse Wave Measurement," Procedia Engineering, vol. 120, pp. 459-463, 2015.

[9] C. Stoeckel, K. Meinel, M. Melzer, and T. Otto, "Thin Film Piezoelectric Aluminum Nitride for Piezoelectric Micromachined Ultrasonic Transducers," in IEEE SENSORS 2018: October 28-31, 2018, Pullman Aerocity, New Delhi, India : 2018 conference proceedings, New Delhi, 2018, pp. 1-4.

[10] G. Rajan and K. Iniewski, Eds., Optical fiber sensors: Advanced techniques and applications. Boca Raton, Fla.: CRC Press, pp. 1-11, 2015.

[11] Y.-J. Rao, Y. Gong, and Z.-L. Ran, Fiber-Optic Fabry-Perot sensors: An introduction. Boca Raton, FL: CRC Press Taylor \& Francis Group, pp. 1-18, 2017.

[12] M. Han, "Theoretical and Experimental Study of Low-Finesse Extrinsic Fabry-Perot Interferometric Fiber Optic Sensors," Dissertation, Virginia Polytechnic Institute and State University, Blacksburg, pp. 21-28, 2006.

[13] M. Friedemann, S. Voigt, M.-L. Werner, R. Hecker, and J. Mehner, "Drift analysis and stabilization of a Fiber Bragg Grating interrogation device," in 25th 2020 International Conference on Applied Electronics: Pilsen, 8-9 September 2020, Pilsen, Czech Republic, 2020, pp. 1-5. 